\title{
IgG4 plasma cell myeloma: new insights into the pathogenesis of IgG4-related disease
}

Julia T Geyer, Ruben Niesvizky, David S Jayabalan, Susan Mathew, Shivakumar Subramaniyam, Alexander I Geyer, Attilio Orazi and Scott A Ely

Department of Pathology and Laboratory Medicine, New York Presbyterian Hospital, Weill Cornell Medical Center, New York, NY, USA

\begin{abstract}
IgG4-related disease is a newly described systemic fibroinflammatory process, characterized by increase in IgG4-positive plasma cells. Its pathogenesis, including the role of IgG4, remains poorly understood. Plasma cell myeloma is typically associated with a large monoclonal serum spike, which is frequently of IgG isotype. We sought to identify and characterize a subset of IgG4-secreting myeloma, as it may provide a biological model of disease with high serum levels of IgG4. Six out of 158 bone marrow biopsies (4\%) from patients with IgG myeloma expressed IgG4. Four patients were men and two were women, with a mean age of 64 (range 53-87) years. Imaging showed fullness of pancreatic head (1), small non-metabolic lymphadenopathy (1), and bone lytic lesions (6). Two patients developed necrotizing fasciitis. All had elevated serum M-protein (mean 2.4, range $0.5-4.2 \mathrm{~g} / \mathrm{dl}$ ), and none had definite signs or symptoms of IgG4-related disease. Four myelomas had plasmablastic morphology. Four had kappa and two had lambda light chain expression. Three cases expressed CD56. Two patients had a complex karyotype. In conclusion, the frequency of IgG4 myeloma correlates with the normal distribution of IgG4 isoform. The patients with IgG4 myeloma appear to have a high rate of plasmablastic morphology and could be predisposed to necrotizing fasciitis. Despite high serum levels of IgG4, none had evidence of IgG4-related disease. These findings suggest that the increased number of IgG4-positive plasma cells is not the primary etiologic agent in IgG4-related disease. Elevated serum levels of IgG4 is not sufficient to produce the typical disease presentation and should not be considered diagnostic of IgG4-related disease. Modern Pathology (2014) 27, 375-381; doi:10.1038/modpathol.2013.159; published online 13 September 2013
\end{abstract}

Keywords: IgG4-related disease; necrotizing fasciitis; plasma cell myeloma; plasmablasts; pathogenesis

IgG4-related disease is a recently described fibroinflammatory condition characterized by mass lesions in various organ systems, a dense lymphoplasmacytic infiltrate rich in IgG4-positive plasma cells, storiform fibrosis, and elevated polyclonal serum IgG4 concentration. ${ }^{1,2}$ Manifestations of IgG4-related disease have been described in virtually every organ system, except in the bone marrow. The symptoms are very sensitive to treatment with steroids. ${ }^{2}$

IgG4 is the rarest IgG isoform and represents $3-6 \%$ of total IgG in the serum of normal subjects. IgG4 differs from other IgG isoforms in the composition of the hinge region, with the resultant formation of bispecific, functionally monovalent antibodies. ${ }^{3-5}$ These antibodies have poor ability to induce

Correspondence: Dr JT Geyer, MD, Division of Hematopathology (Starr 711A), Department of Pathology and Laboratory Medicine, New York Presbyterian Hospital, Weill Cornell Medical Center, 525 East 68th Street, New York, NY 10065, USA.

E-mail: jut9021@med.cornell.edu

This work was presented in part at the 102nd Annual Meeting of the United States and Canadian Academy of Pathology.

Received 8 May 2013; revised 9 July 2013; accepted 14 July 2013; published online 13 September 2013 complement, activate cells, and crosslink identical antigens. ${ }^{4}$ A classic study of beekeepers showed that during the first 6 months of exposure, most antibodies to bee venom were IgG1; however, with continued stimulation IgG1 titers stabilized, whereas IgG4 titers continued to rise (up to $\sim 90 \%$ of the response). ${ }^{6}$ A mixture of early and late antibodies showed no evidence of immunoprecipitation. Thus, IgG4 may have predominantly anti-inflammatory activity and provide protection against pathogenic antibodies in autoimmune disease. ${ }^{6}$

The pathogenesis of IgG4-related disease is unknown but is likely related to either autoimmunity or chronic infection. ${ }^{1}$ There is an activation of type 2 helper T cells with increase in IL-2, IL-4, IL-5, IL-10, IL-13, and TGF- $\beta$, leading to recruitment of IgG4positive plasma cells, B-lymphocytes, eosinophils, and fibroblasts. ${ }^{1,7,8}$

The prototype of an IgG4-related disease is autoimmune pancreatitis, which was initially recognized in the early 1960s. ${ }^{9}$ In recent years, these patients have been extensively studied and were found to have additional involvement of numerous extrapancreatic sites, such as the ocular adnexa, 
salivary glands, kidney, lung, liver, gallbladder, and lymph nodes. ${ }^{10-16}$ On the other hand, some patients may present with primary extrapancreatic disease and have no evidence of autoimmune pancreatitis. ${ }^{17,18}$ Patients may have involvement of one organ or have multiorgan disease either at diagnosis or metachronously. Up to $40 \%$ have evidence of allergic disease.

Serum IgG4 elevation is a diagnostic marker of IgG-related disease. ${ }^{19,20}$ At the same time, IgG4 elevation may also be associated with various unrelated disorders, such as repeated infections, autoimmune disease, allergic conditions, carcinoma, cystic fibrosis with concomitant Pseudomonas aeruginosa, and multicentric Castleman disease. ${ }^{21-23}$ However, it appears that the serum level of IgG4 is consistently lower in these conditions compared with IgG4-related disease $(>135 \mathrm{mg} / \mathrm{dL}) .21-23$

The diagnostic criteria for IgG4-related disease are still evolving. Two recently proposed classifications are based on elevated levels of serum IgG4 ( $>135 \mathrm{mg} / \mathrm{dl}$ or $>1.5$ times the normal upper limit); enlargement, mass lesions, or dysfunction in one or more characteristic organs; typical radiographic features; and the histologic presence of a prominent lymphoplasmacytic infiltrate, abundant IgG4-positive plasma cells, storiform swirling fibrosis, and obliterative phlebitis. , $^{24,25}$

Patients with IgG4-related disease have a 3.5 times higher risk of cancer than the general population. ${ }^{26}$ Reported cases consist predominantly of extranodal marginal zone lymphoma (MALT lymphoma), which may either arise in the background of a preexisting non-clonal IgG4-related chronic sclerosing lesion or have evidence of plasmacytic differentiation with IgG4-positive monoclonal plasma cells. ${ }^{27-30}$ Cases of salivary duct carcinoma arising in the background of IgG4-associated sialadenitis and pancreatic carcinoma associated with autoimmune pancreatitis have also been reported. ${ }^{31,32}$

In summary, the role of IgG4 in the pathogenesis of IgG4-related disease is currently unknown. Plasma cells in myeloma patients are mature immunocompetent cells that have undergone somatic hypermutation and class switch recombination. ${ }^{33}$ Most malignant plasma cells secrete antibodies coded by functional VDJh rearrangements, detected as paraprotein. ${ }^{33}$ We sought to characterize a population of secretory IgG4-positive plasma cell myeloma. The goals of the study are to verify the incidence of IgG4 myeloma, determine its clinicopathologic features, and investigate for possible association of IgG4 myeloma with signs or symptoms of pre-existing, concomitant, or emerging IgG4related disease.

\section{Materials and methods}

A computer-assisted search of the Weill Cornell Myeloma Center Database of the Weill Cornell
Medical College/New York Presbyterian Hospital and review of the identified cases disclosed 158 consecutive bone marrow biopsies from patients with IgG myeloma. The bone marrow biopsies, aspirates, and peripheral blood smears (when available) were reviewed. Plasmablastic morphology was defined as enlarged plasma cells with fine chromatin pattern, prominent central nucleolus, and high nuclear-to-cytoplasmic ratio. Immunohistochemical staining for IgG4 (Cell Marque Clone MRQ-44, prediluted) was performed on all cases and six positive cases were identified. These cases had further immunohistochemical staining for kappa (Dako A0191, (1:10 000)) and lambda (Dako A0193, (1:20 000)) immunoglobulin light chains, CD56 (Leica NCL-CD56-1B6, (1:10)) and double staining for Cyclin D1/CD138 (Serotec/Thermo Sci MCA2459/RM-9104 S, (1:200)/(1:10)), Cyclin D3/ CD138 (Serotec/Leica MCA2459/NCL-CYCLIN D3, $(1: 200) /(1: 10))$, and Ki-67/CD138 (Serotec/Dako MCA2459/M7240, (1:200)/(1:50)). Immunohistochemical staining was accomplished using the Bond III Autostainer (Leica Microsystems, IL, USA). Antigen retrieval was followed by heating the sections at $37^{\circ} \mathrm{C}$ in Bond Enzyme Solution (Leica Microsystems) for $10 \mathrm{~min}$.

All additional surgical pathology and immunopathology materials were retrieved and reviewed for the six IgG4-positive myeloma cases. Clinical information, follow-up, and treatment information were acquired by review of the clinical charts following institutional review board approval. Cytogenetic studies were performed on overnight unstimulated bone marrow cultures. Cells were harvested and G-banded. When possible, a total of 20 metaphases were evaluated. The findings were described according to the 2009 International System for Human Cytogenetic Nomenclature. ${ }^{34}$ FISH was carried out using the LSI FGFR3 (Spectrum Orange - SO) - IGH (Spectrum Green - SG) dual color dual fusion probes, LSI CCND1/MYEOV (SO) IGH (SG) dual color dual fusion probes, LSI 13 (SG), and LSI TP53 (SO) probes (Vysis/Abbott Molecular, Des Plaines, IL, USA) to rule out the translocations $\mathrm{t}(4 ; 14)(\mathrm{p} 16 ; \mathrm{q} 32)$ and $\mathrm{t}(11 ; 14)(\mathrm{q} 13 ; \mathrm{q} 32)$ and deletions of $13 \mathrm{q} 14$ region and TP53 gene, respectively. FISH was also performed using the LSI p58/LSI Telomere 1p/LSI 1q25, D5S23/D5S721, CEP 9, and CEP 15 probes to evaluate for chromosome 1 abnormalities and trisomy of chromosomes 5, 9, and 15, respectively. Two hundred interphase nuclei were evaluated for each probe/probe set.

\section{Results}

Six out of $158(4 \%)$ cases of IgG-positive myeloma had extensive expression of IgG4 by the clonal plasma cells. The clinical, morphologic, immunohistochemical, and cytogenetic findings are 
presented in Table 1 and Table 2 and are summarized below.

\section{Clinical Information}

There were four male and two female subjects, with a mean age of 64 (range 53-87) years. The initial diagnosis was monoclonal gammopathy of undetermined significance in one patient (transformed to myeloma in 5 years), smoldering myeloma in one patient (transformed to symptomatic myeloma in 2 years), and active myeloma in the remaining four patients. All patients had elevated serum M-protein at presentation (mean 2.4, range 0.5-4.2 g/dl). Each patient's highest median M-spike was 3.1, with a range of 1.2 up to $4.9 \mathrm{~g} / \mathrm{dl}$. All but one patient were treated with multiple courses of chemotherapy. Four patients underwent autologous bone marrow transplants; two of them had two transplants. One patient developed therapy-related myelodysplastic syndrome 5 years after initial presentation. Four patients died after a median follow-up of 4.5 years (range, 1 month to 11 years), whereas two patients are alive with disease 5 and 11 years after the initial diagnosis.

Two patients had possible signs and symptoms of IgG4-related disease. One patient, a 53-year-old man, had a long-standing history of chronic sinusitis and small non-metabolic lymphadenopathy on the initial PET-CT scan. He was diagnosed with plasma cell myeloma during preoperative work-up for sinus surgery (patient 1, see Table 1 and Table 2). Another patient, a 63-year-old woman, had abdominal CT imaging 11 years after initial diagnosis, which showed a mild fullness of the pancreatic head without associated biliary ductal dilatation or main pancreatic duct narrowing. A concurrent abdominal ultrasound had normal findings. There were no laboratory or clinical signs of pancreatitis (patient 5 , see Table 1 and Table 2). Unusually, both patients developed life-threatening episodes of necrotizing fasciitis (due to Streptococcus pyogenes and Escherichia coli), which occurred immediately following a cycle of chemotherapy (doxorubicin, vincristine, and dexamethasone or dexamethasone, cyclophosphamide, etoposide, and cisplatin) in the setting of active myeloma. ${ }^{35}$ Patients' M-protein was 2.85 and $1.6 \mathrm{~g} / \mathrm{dl}$, respectively, at the time of the infection. Patient 1 recovered following three debridement surgeries, development of osteomyelitis, and a long hospital stay. Patient 5 also underwent three surgeries accompanied by a rapidly increasing M-protein (1.6-3.2 g/dl) and increasing pancytopenia. She died 1 month after the presentation with necrotizing fasciitis.

\section{Pathological and Immunohistochemical Findings}

All bone marrow biopsies had an increased number of plasma cells (Figure 1). Three of six cases had evidence of plasmablastic morphology at presentation (Figures 1a, d and e); one of these cases also had marked plasma cell atypia (Figure 1e). One additional plasma cell myeloma acquired plasmablastic features 5 years following initial diagnosis, accompanied by increased proliferation rate and a new

Table 1 Summary of the clinical information

\begin{tabular}{|c|c|c|c|c|c|c|c|}
\hline & Age/sex & Follow-up & $\begin{array}{l}\text { Clinical } \\
\text { presentation }\end{array}$ & Imaging & M-protein & Plasmacytoma & $N F$ \\
\hline 1 & $56 / \mathrm{M}$ & 3y (DOD) & Chronic sinusitis & LAN & $3.6 \mathrm{~g} / \mathrm{dl}$ & No & Yes $(\times 3)$ \\
\hline 2 & $61 / \mathrm{M}$ & 6y (DOD) & Chest pain & LL & $0.9 \mathrm{~g} / \mathrm{dl}$ & Yes & No \\
\hline 3 & $87 / \mathrm{M}$ & $1 \mathrm{~m}$ (DOD) & Cord compression & LL & $2.3 \mathrm{~g} / \mathrm{dl}$ & Yes & No \\
\hline 4 & $61 / \mathrm{F}$ & $5 y$ & Smoldering myeloma & Normal & $2.8 \mathrm{~g} / \mathrm{dl}$ & Yes & No \\
\hline 5 & $63 / \mathrm{F}$ & 11y (DOD) & MGUS, back pain & Pancreas LL & $0.5 \mathrm{~g} / \mathrm{dl}$ & Yes & Yes $(\times 2)$ \\
\hline 6 & $53 / \mathrm{M}$ & $11 \mathrm{y}$ & Pain & Normal & $4.2 \mathrm{~g} / \mathrm{dl}$ & No & No \\
\hline
\end{tabular}

Abbreviations: DOD, dead of disease; MGUS, monoclonal gammopathy of undetermined significance; LAN, lymphadenopathy; LL, lytic lesions; $\mathrm{NF}$, necrotizing fasciitis.

Table 2 Summary of histological, immumohistochemical, and cytogenetic findings at presentation

\begin{tabular}{|c|c|c|c|c|c|c|c|}
\hline & Fibrosis & Plasmablasts & Ki-67 PR & CD56 & $B C L-1$ & Karyotype & FISH \\
\hline 1 & $1+$ & Yes & $22 \%$ & + & + & Normal & $\operatorname{del}(13 q), H$ \\
\hline 2 & $1+$ & No & $<1 \%$ & - & - & Normal & Normal \\
\hline 3 & $3+$ & No & $9 \%$ & - & + & Normal & $\mathrm{t}(11 ; 14), \mathrm{H}$ \\
\hline 4 & $2+$ & Yes & $2 \%$ & + & - & Complex & $\mathrm{H}$ \\
\hline 5 & $1+$ & Yes & $74 \%$ & + & - & Complex & $\begin{array}{l}\mathrm{t}(4 ; 14), \operatorname{del}(17 p), \\
1 \mathrm{q} 21 \text { gain, } \mathrm{H}\end{array}$ \\
\hline 6 & $1+$ & No & $2 \%$ & - & + & Normal & Normal \\
\hline
\end{tabular}

Abbreviations: H, hyperdiploidy; PR, proliferation rate. 

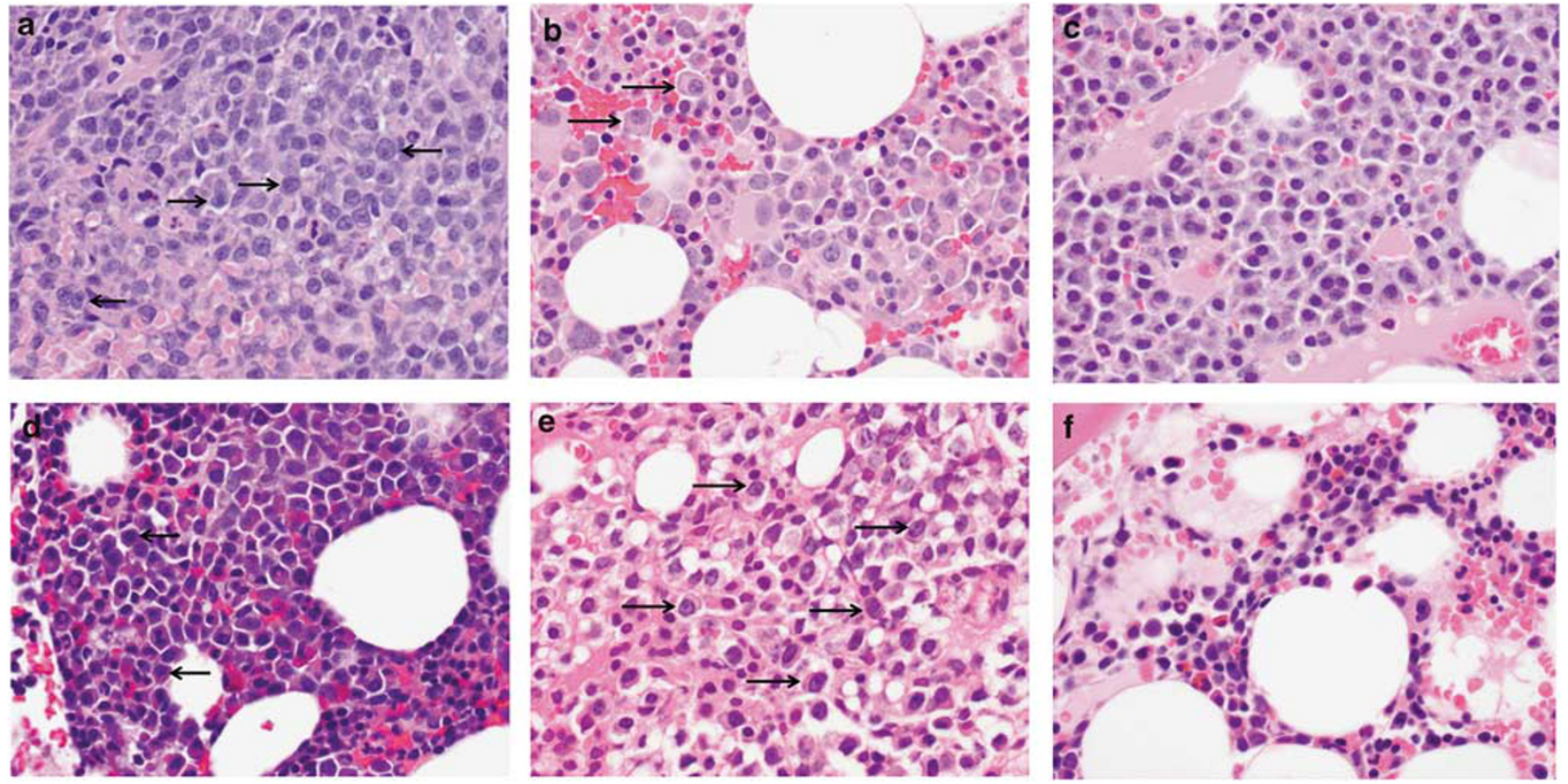

Figure 1 IgG4 myeloma morphology. (a-f): representative images from patients 1 through 6, respectively (H\&E, bone marrow biopsy, original magnification $\times 400$ ). Arrows indicate plasmablasts in cases $1,2,4$, and 5 .

complex karyotype (patient 2, Figure 1b). One plasma cell myeloma had numerous Dutcher bodies (Figure 1d). Two of the six cases had evidence of increased reticulin fibrosis in the bone marrow. Four plasma cell myelomas expressed monotypic kappa immunoglobulin light chain and two cases expressed monotypic lambda light chain. Three plasma cell myelomas aberrantly expressed CD56 or Cyclin D1. One of the six cases was positive for both CD56 and Cyclin D1. All cases were negative for Cyclin D3. With Ki-67/CD138 immunohistochemical double staining, the proliferative fraction of the plasma cells ranged from $<1$ to $74 \%$ (mean, $25 \%$ ).

Three of the six patients had several bone marrow biopsies available for review (3-4 biopsies/case, spanning 3-6 years). All biopsies with evidence of myeloma had numerous IgG4-positive plasma cells (Figure 2). Negative/normal bone marrow biopsies did not have an increased number of IgG4-positive plasma cells. Both patients with necrotizing fasciitis underwent debridement surgery. These skin and soft tissue biopsies contained extensive necrosis and an acute inflammatory infiltrate, with no evidence of IgG4-positive plasma cells.

\section{Cytogenetic Results}

At diagnosis, four of the six patients had cytogenetic abnormalities: two had a complex karyotype and two had a normal karyotype with abnormal interphase FISH and evidence of hyperdiploidy. One patient with initially normal karyotype and FISH developed a hyperdiploid genotype using FISH 3 years after diagnosis, followed by a complex

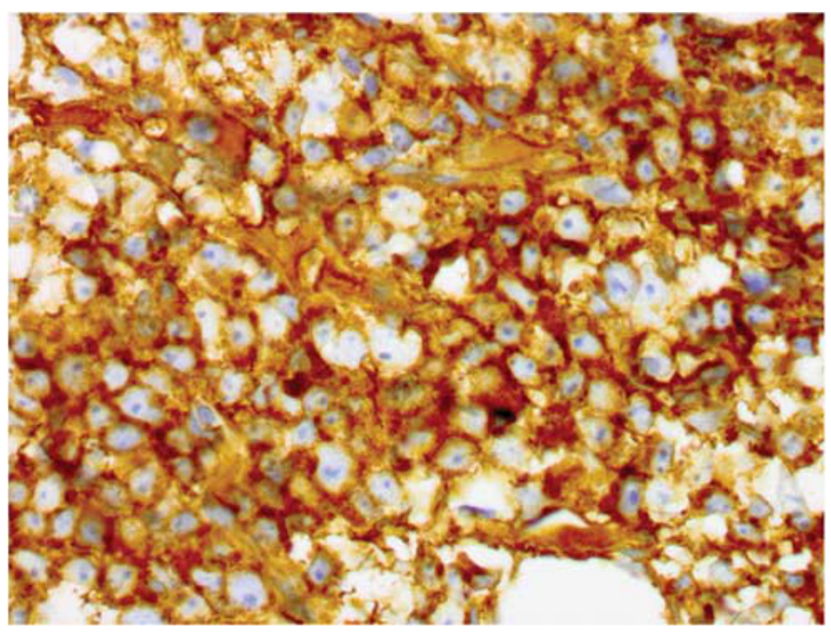

Figure 2 Strong diffuse immunohistochemical staining with IgG4 (patient 5, original magnification $\times 400$ ).

karyotype with the evidence of monosomy of chromosomes 5, 7, 9, and 20 and partial loss of 12p 1 year later (patient 2). At the same time, this patient was diagnosed with a high-grade myelodysplastic syndrome. Cell sorting was not performed; however, as these cytogenetic abnormalities are common in myelodysplastic syndrome and rare in plasma cell myeloma, they likely correspond to the myeloid clone and not plasma cells.

\section{Discussion}

In this study, IgG4 myeloma represented 4\% (6/158) of the IgG myeloma cases. The frequency of IgG4 
myeloma correlates with the normal distribution of the IgG4 isoform (3-6\% of IgG in healthy subjects). Studies of subclass distribution in IgG monoclonal gammopathy of undetermined significance and myeloma have last been performed in the 1980s and showed similar data, with IgG4 representing 2$8 \%$ of IgG plasma cell neoplasms. ${ }^{36-39}$ All patients in this study had persistently high levels of IgG4 paraprotein (average, $2.4 \mathrm{~g} / \mathrm{dl}$ ). Two patients had a documented phase of monoclonal gammopathy of undetermined significance and smoldering myeloma. Based on the current knowledge of myeloma biology, it can be assumed that the remaining patients also had a prodromal asymptomatic stage with gradually increasing levels of IgG4 paraprotein. Clinical course of plasma cell myeloma is characterized by disease relapses and exacerbations with fluctuating levels of paraprotein. Thus, we hypothesized that patients with IgG4 myeloma may offer an opportunity to study the long-term effects of high-concentration of serum IgG4.

The patients with IgG4 myeloma appear to have typical clinical, laboratory, and morphologic features of IgG myeloma with two exceptions. The proportion of cases with plasmablastic morphology is increased compared with the reported literature, at $67 \%$ of the cases vs $5-15 \%$ in the previous studies. ${ }^{40-43}$ The definition of plasmablastic myeloma is controversial. Bartl et $a I^{43}$ required plasmablasts to represent the predominant cell type in the bone marrow biopsy and defined plasmablasts as having large, immunoblast-like nucleus with a prominent nucleolus and a moderate amount of basophilic cytoplasm. In contrast, Greipp et al $1^{40,42}$ established criteria based on examination of bone marrow aspirates and required $>2 \%$ plasmablasts, which were described as having large centrally placed nucleus $(>10 \mu \mathrm{m}$ in diameter $)$ or a nucleolus ( $>2 \mu \mathrm{m}$ in diameter), a high nuclear-to-cytoplasmic ratio, and lack of a perinuclear hof. Not all of the cases in this study had a bone marrow aspirate; thus, a more inclusive definition of plasmablasts was used. The significance of plasmablastic morphology in patients treated with the new antimyeloma drugs is unclear; however, several older studies showed a worse response to treatment and higher rate of progression in these patients. ${ }^{40,42,43}$ The patients in this study did not appear to do significantly worse than other patients treated at our institution.

Another unusual feature of IgG4 myeloma is the presence of necrotizing fasciitis in two patients. This association is so rare that these patients had been a subject of a prior case report by our group. ${ }^{35}$ In addition to these patients, only two other cases have been described in the literature. ${ }^{44,45}$ Known risk factors of necrotizing fasciitis include NSAID use, diabetes, intravenous drug abuse, obesity, recent surgery, trauma, and immunosuppression. Both patients' episodes of necrotizing fasciitis occurred in the setting of active myeloma immediately following a cycle of chemotherapy. Thus, we hypothesize that as IgG4 appears to have an immunosuppressive effect, high serum levels of IgG4 in combination with high-dose dexamethasone used for chemotherapy purposes may create a unique host susceptibility to bacterial soft tissue infection. This association is clinically important because of the fulminant course of untreated necrotizing fasciitis, with high ensuing morbidity and mortality.

CD56 expression was seen in $50 \%$ of the cases, similar to $70 \%$ reported in the literature. ${ }^{46}$ All cases of CD56-positive myeloma had a complex karyotype. Lack of CD56 expression in plasma cell myeloma has previously been associated with worse outcome; however, recent studies of patients treated with high-dose chemotherapy did not find a significant difference in patient survival. ${ }^{47,48}$ Cytogenetic analysis detected chromosomal abnormalities in 5/6 cases $(83 \%)$, similar to $90 \%$ of all cases of newly diagnosed myeloma. ${ }^{46}$ Two patients had a complex karyotype at presentation, including one with three high-risk abnormalities (translocation $t(4 ; 14)$, gain of the 1q21 region and deletion 17p) - findings that are associated with unfavorable outcome and shorter median survival - whereas two patients had evidence of trisomies of odd-numbered chromosomes (hyperdiploidy) that are typically associated with better survival. ${ }^{49,50}$ This distribution of genetic abnormalities is similar to the reported literature and does not indicate a unique subtype of plasma cell myeloma. ${ }^{46,49,50}$

Aside from large numbers of IgG4 + plasma cells, the histological features of bone marrows and soft tissue debridement specimens did not show any characteristics of IgG4-related disease, such as lymphoplasmacytic inflammation, lymphoid follicles with expanded germinal centers, obliterative phlebitis, or prominent cellular fibrosis, composed of activated fibroblasts, lymphocytes, and plasma cells. ${ }^{10,20,51-58}$ Despite sustained high serum levels of IgG4, none of the patients fulfilled diagnostic criteria for IgG4-related systemic disease. One patient had a history of chronic sinusitis and small lymphadenopathy, which are relatively nonspecific. Another patient had an incidental finding of mild fullness of the pancreatic head on CT imaging but no other signs or symptoms of pancreatitis. ${ }^{54}$

In summary, our findings suggest that neither an increased number of IgG4-positive plasma cells nor a high sustained serum IgG4 is the primary etiologic agent in IgG4-related disease. Elevated serum level of IgG4 is probably not sufficient to produce the typical disease presentation and should not be considered diagnostic of IgG4-related disease. IgG4 myeloma represents $\sim 4 \%$ of IgG-positive plasma cell myeloma and may represent a unique subset with plasmablastic morphology and frequent association with necrotizing fasciitis. IgG4 myeloma should be considered in the differential diagnosis of patients with high serum levels of IgG4. 


\section{Disclosure/conflict of interest}

The authors declare no conflict of interest.

\section{References}

1 Stone JH, Zen Y, Deshpande V. IgG4-related disease. N Engl J Med 2012;366:539-551.

2 Carruthers MN, Stone JH, Khosroshahi A. The latest on IgG4-RD: a rapidly emerging disease. Curr Opin Rheumatol 2012;24:60-69.

3 Aalberse RC, Schuurman J. IgG4 breaking the rules. Immunology 2002;105:9-19.

4 van der Neut Kolfschoten M, Schuurman J, Losen M, et al. Anti-inflammatory activity of human IgG4 antibodies by dynamic Fab arm exchange. Science 2007;317:1554-1557.

5 Aalberse RC, Stapel SO, Schuurman J, et al. Immunoglobulin G4: an odd antibody. Clin Exp Allergy 2009;39:469-477.

6 Aalberse RC, van der Gaag R, van Leeuwen J. Serologic aspects of IgG4 antibodies. I. Prolonged immunization results in an IgG4-restricted response. J Immunol 1983;130:722-726.

7 Genton C, Wang Y, Izui S, et al. The Th2 lymphoproliferation developing in LatY136F mutant mice triggers polyclonal B cell activation and systemic autoimmunity. J Immunol 2006;177:2285-2293.

8 Rubin LA, Nelson DL. The soluble interleukin-2 receptor: biology, function, and clinical application. Ann Intern Med 1990;113:619-627.

9 Gohring G, Giagounidis A, Busche G, et al. Patients with del(5q) MDS who fail to achieve sustained erythroid or cytogenetic remission after treatment with lenalidomide have an increased risk for clonal evolution and AML progression. Ann Hematol 2010;89: 365-374.

10 Hamano H, Arakura N, Muraki T, et al. Prevalence and distribution of extrapancreatic lesions complicating autoimmune pancreatitis. J Gastroenterol 2006;41: 1197-1205.

11 Deshpande V, Mino-Kenudson M, Brugge W, et al. Autoimmune pancreatitis: more than just a pancreatic disease? A contemporary review of its pathology. Arch Pathol Lab Med 2005;129:1148-1154.

12 Cheuk W, Yuen HK, Chan JK. Chronic sclerosing dacryoadenitis: part of the spectrum of IgG4-related Sclerosing disease? Am J Surg Pathol 2007;31:643-645.

13 Cornell LD, Chicano SL, Deshpande V, et al. Pseudotumors due to IgG4 immune-complex tubulointerstitial nephritis associated with autoimmune pancreatocentric disease. Am J Surg Pathol 2007;31:1586-1597.

14 Zen Y, Fujii T, Harada K, et al. Th2 and regulatory immune reactions are increased in immunoglobin G4related sclerosing pancreatitis and cholangitis. Hepatology 2007;45:1538-1546.

15 Masaki Y, Dong L, Kurose N, et al. Proposal for a new clinical entity, IgG4-positive multiorgan lymphoproliferative syndrome: analysis of 64 cases of IgG4-related disorders. Ann Rheum Dis 2009;68:1310-1315.

16 Yamamoto M, Takahashi H, Ohara M, et al. A new conceptualization for Mikulicz's disease as an IgG4related plasmacytic disease. Mod Rheumatol 2006;16: 335-340.
17 Ghazale A, Chari ST, Zhang L, et al. Immunoglobulin G4-associated cholangitis: clinical profile and response to therapy. Gastroenterology 2008;134:706-715.

18 Cheuk W, Yuen HK, Chu SY, et al. Lymphadenopathy of IgG4-related sclerosing disease. Am J Surg Pathol 2008;32:671-681.

19 Hamano H, Kawa S, Horiuchi A, et al. High serum IgG4 concentrations in patients with sclerosing pancreatitis. N Engl J Med 2001;344:732-738.

20 Deshpande V, Chicano S, Finkelberg D, et al. Autoimmune pancreatitis: a systemic immune complex mediated disease. Am J Surg Pathol 2006;30: 1537-1545.

21 Ebbo M, Grados A, Bernit E, et al. Pathologies associated with serum IgG4 elevation. Int J Rheumatol 2012;2012:602809.

22 Ryu JH, Horie R, Sekiguchi $\mathrm{H}$, et al. Spectrum of disorders associated with elevated serum IgG4 levels encountered in clinical practice. Int J Rheumatol 2012;2012:232960.

23 Yamamoto M, Tabeya T, Naishiro Y, et al. Value of serum IgG4 in the diagnosis of IgG4-related disease and in differentiation from rheumatic diseases and other diseases. Mod Rheumatol 2012;22:419-425.

24 Okazaki K, Uchida K, Koyabu M, et al. Recent advances in the concept and diagnosis of autoimmune pancreatitis and IgG4-related disease. J Gastroenterol 2011:46:277-288.

25 Okazaki K, Uchida K, Miyoshi $\mathrm{H}$, et al. Recent concepts of autoimmune pancreatitis and IgG4-related disease. Clin Rev Allergy Immunol 2011;41:126-138.

26 Yamamoto M, Takahashi H, Tabeya T, et al. Risk of malignancies in IgG4-related disease. Mod Rheumatol 2012;22:414-418.

27 Kim T, Grobmyer SR, Dixon LR, et al. Autoimmune pancreatitis and concurrent small lymphocytic lymphoma: not just a coincidence? J Gastrointest Surg 2008;12:1566-1570.

28 Cheuk W, Yuen HK, Chan AC, et al. Ocular adnexal lymphoma associated with IgG4 + chronic sclerosing dacryoadenitis: a previously undescribed complication of IgG4-related sclerosing disease. Am J Surg Pathol 2008;32:1159-1167.

29 Takahashi N, Ghazale AH, Smyrk TC, et al. Possible association between IgG4-associated systemic disease with or without autoimmune pancreatitis and nonHodgkin lymphoma. Pancreas 2009;38:523-526.

30 Venkataraman G, Rizzo KA, Chavez JJ, et al. Marginal zone lymphomas involving meningeal dura: possible link to IgG4-related diseases. Mod Pathol 2011;24: 355-366.

31 Gill J, Angelo N, Yeong ML, et al. Salivary duct carcinoma arising in IgG4-related autoimmune disease of the parotid gland. Hum Pathol 2009;40:881-886.

32 Gupta R, Khosroshahi A, Shinagare S, et al. Does autoimmune pancreatitis increase the risk of pancreatic carcinoma? A retrospective analysis of pancreatic resections. Pancreas 2013;42:506-510.

33 Gonzalez D, van der Burg M, Garcia-Sanz R, et al. Immunoglobulin gene rearrangements and the pathogenesis of multiple myeloma. Blood 2007;110:3112-3121.

34 ISCN 2009 An international System for Human Cytogenetic Nomenclature. S Karger Publishing: Basel, 2009.

35 Sultan S, Morawa E, Niesvizky R, et al. Necrotizing soft tissue infection in two patients with multiple myeloma. Surg Infect (Larchmt) 2011;12:391-395. 
36 Aucouturier P, Preud'Homme JL. Subclass distribution of human myeloma proteins as determined with monoclonal antibodies. Immunol Lett 1987;16:55-57.

37 Papadea C, Reimer CB, Check IJ. IgG subclass distribution in patients with multiple myeloma or with monoclonal gammopathy of undetermined significance. Ann Clin Lab Sci 1989;19:27-37.

38 Tichy M, Hrncir Z, Mracek J. Subclasses IgG1-IgG4 in 84 sera with IgG paraprotein. Neoplasma 1978;25: 107-110.

39 Kyle RA, Gleich GJ. IgG subclasses in monoclonal gammopathy of undetermined significance. J Lab Clin Med 1982;100:806-814.

40 Greipp PR, Raymond NM, Kyle RA, et al. Multiple myeloma: significance of plasmablastic subtype in morphological classification. Blood 1985;65: 305-310.

41 Carter A, Hocherman I, Linn S, et al. Prognostic significance of plasma cell morphology in multiple myeloma. Cancer 1987;60:1060-1065.

42 Greipp PR, Leong T, Bennett JM, et al. Plasmablastic morphology-an independent prognostic factor with clinical and laboratory correlates: Eastern Cooperative Oncology Group (ECOG) myeloma trial E9486 report by the ECOG Myeloma Laboratory Group. Blood 1998;91:2501-2507.

43 Bartl R, Frisch B, Fateh-Moghadam A, et al. Histologic classification and staging of multiple myeloma. A retrospective and prospective study of 674 cases. Am J Clin Pathol 1987;87:342-355.

44 Rosser A, Swallow G, Swann RA, et al. Salmonella enteritidis necrotising fasciitis in a multiple myeloma patient receiving bortezomib. Int J Hematol 2010; 91:149-151.

45 Kanoh T. [Multiple myeloma complicated by necrotizing fasciitis]. Rinsho Ketsueki 1996;37:1309-1313.

46 McKenna RW, Kyle RA, Kuehl WM, et al. Plasma cell neoplasms, In: Swerdlow S, Campo E, Harris NL, Jaffe ES, Pileri SA, Stein H, Thiele J, Vardiman JW(eds) WHO Classification of Tumours of Haematopoietic and Lymphoid Tissues. IARC: Lyon, 2008.

47 Hundemer M, Klein U, Hose D, et al. Lack of CD56 expression on myeloma cells is not a marker for poor prognosis in patients treated by high-dose chemotherapy and is associated with translocation $\mathrm{t}(11 ; 14)$. Bone Marrow Transplant 2007;40:1033-1037.

48 Chang H, Samiee S, Yi QL. Prognostic relevance of CD56 expression in multiple myeloma: a study including 107 cases treated with high-dose melphalan-based chemotherapy and autologous stem cell transplant. Leuk Lymphoma 2006;47:43-47.

49 Nemec P, Zemanova Z, Kuglik P, et al. Complex karyotype and translocation $\mathrm{t}(4 ; 14)$ define patients with high-risk newly diagnosed multiple myeloma: results of CMG2002 trial. Leuk Lymphoma 2012;53: 920-927.

50 Kumar S, Fonseca R, Ketterling RP, et al. Trisomies in multiple myeloma: impact on survival in patients with high-risk cytogenetics. Blood 2012;119:2100-2105.

51 Chan JK. Kuttner tumor (chronic sclerosing sialadenitis) of the submandibular gland: an underrecognized entity. Adv Anat Pathol 1998;5:239-251.

52 Kitagawa S, Zen Y, Harada K, et al. Abundant IgG4positive plasma cell infiltration characterizes chronic sclerosing sialadenitis (Kuttner's tumor). Am J Surg Pathol 2005;29:783-791.

53 Geyer JT, Ferry JA, Harris NL, et al. Chronic sclerosing sialadenitis (Kuttner tumor) is an IgG4-associated disease. Am J Surg Pathol 2010;34:202-210.

54 Finkelberg DL, Sahani D, Deshpande V, et al. Autoimmune pancreatitis. N Engl J Med 2006;355: 2670-2676.

55 Kamisawa T, Nakajima H, Egawa N, et al. IgG4-related sclerosing disease incorporating sclerosing pancreatitis, cholangitis, sialadenitis and retroperitoneal fibrosis with lymphadenopathy. Pancreatology 2006;6: 132-137.

56 Masaki Y, Sugai S, Umehara H. IgG4-related diseases including Mikulicz's disease and sclerosing pancreatitis: diagnostic insights. J Rheumatol 2010;37: 1380-1385.

57 Takano KI, Yamamoto M, Takahashi H, et al. Clinicopathologic similarities between Mikulicz disease and Kuttner tumor. Am J Otolaryngol 2009;31:429-434.

58 Yamamoto M, Harada S, Ohara M, et al. Clinical and pathological differences between Mikulicz's disease and Sjogren's syndrome. Rheumatology (Oxford) 2005;44:227-234. 